\title{
Outcome of deceased donor renal transplantation in Mongolia: a single-center experience
}

\author{
Lkhaakhuu Od-Erdene, Tseren Khishgee, Dagvadorj Bayan-Undur, Donkhim Chuluunbaatar, Batsuuri Batsaikhan, \\ Jigjidsuren Sarantsetseg
}

Organ transplantation Center, First Central Hospital of Mongolia, Ulaanbaatar, Mongolia

Background: Renal transplantation (RTx) has now become an accepted therapeutic modality of choice for end-stage renal disease (ESRD) patients in Mongolia. In last 5 years very fast developed organ transplantation system in Mongolia. In this time we can increase number of RTx from deceased donors in Mongolia. This single-center study was to evaluate the outcome of deceased donors RTx in ESRD patients.

Methods: We analyzed the outcome of 223 RTx (195 living donor and 29 deceased donors in ESRD patients at the time of transplantation between 2008 and 2020. Approval by the Internal Review Board and written informed consent from all patients were obtained.

Results: A total of 29 deceased donors at our center: the mean donor age was 47.2 years and 41.4 years in the recipients. Male recipients constituted $82.7 \%$ and $46.6 \%$ in deceased donors. In deceased donor RTx, 1-, 3- and 5-year patient survival was $93.2 \%$, $89.6 \%$ and $86.2 \%$ respectively and graft survival in $1,3,5$ and 10 years was $96.5 \%, 89.6 \%, 89.6 \%$, and $82.8 \%$ respectively. Induction immunosuppressant /IS/ was Basiliximab with steroid for all case and posttransplant maintenance consisted of calcineurin inhibitor-based regimen and MMF with low dose steroid. Cause of ESRD was $79.4 \%$ chronic glomerulonephritis, $6.8 \%$ diabetic mellitus type 2, 3.5\% polycystic kidney disease and urine system abnormality. Mean duration dialysis treatment time for recipients was 39.9 months. Percentage of dialysis modality was PD 17.3\% and HD 82.7\%. Complication was TCMR one case, infection complication included urine tract, herpes simples virus, $\mathrm{H} 1 \mathrm{~N} 1$, hepatitis $\mathrm{C}$ virus, and hepatitis $\mathrm{B}$ virus. Patient death cause was heart failure, respiratory infection, suicide, traffic accident.

Conclusions: Deceased donor RTx in ESRD patients has acceptable patient and graft survival in Mongolia. But the number of deceased donors is still low. We need develop system of organ donation. 